\title{
Extensor tendon release in tennis elbow: results and prognostic factors in 80 elbows
}

\author{
Eirik Solheim $\cdot$ Janne Hegna $\cdot$ Jannike Øyen
}

Received: 14 June 2010/Accepted: 24 February 2011/Published online: 16 March 2011

(C) The Author(s) 2011. This article is published with open access at Springerlink.com

\begin{abstract}
Purpose The objectives of this study were to evaluate the results in the outpatient treatment of recalcitrant lateral epicondylitis with release of the common extensor origin according to Hohmann and to determine any prognostic factors.

Methods Eighty tennis elbows in 77 patients with a characteristic history of activity-related pain at the lateral epicondyle interfering with the activities of daily living refractory to conservative care for at least 6 months and a confirmatory physical examination were included. Clinical outcome was evaluated using the QuickDASH score system. Data were collected before the operation and at the medians of 18 months (range 6-36 months; short term) and 4 years (range 3-6 years; medium term) postoperatively.

Results The mean QuickDASH was improved both at the short- and the medium-term follow-ups and did not change significantly between the follow-ups. At the final followup, the QuickDASH was improved in 78 out of 80 elbows and $81 \%$ was rated as excellent or good (QuickDASH $<40$ points). We found a weak correlation between residual symptoms (a high QuickDASH score) at the final follow-up and high level of baseline symptoms $(r=0.388)$, acute occurrence of symptoms $(r=0.362)$, long duration of
\end{abstract}

E. Solheim · J. Hegna $\cdot$ J. Øyen

Bergen Surgical Hospital, Bergen, Norway

E. Solheim $(\bowtie)$

Department of Orthopaedics, Deaconess University Hospital,

Haraldsplass, 5009 Bergen, Norway

e-mail: eirik.solheim@uib.no

E. Solheim · J. Øyen

Department of Surgical Sciences, Faculty of Medicine and

Dentistry, University of Bergen, Bergen, Norway symptoms $(r=0.276)$, female gender $(r=0.269)$ and young age $(r=0.203)$, whereas occurrence in dominant arm, a work-related cause or strenuous work did not correlate significantly with the outcome.

Conclusion Open lateral extensor release performed as outpatient surgery results in improved clinical outcome at both short- and medium-term follow-ups with few complications. High baseline disability, sudden occurrence of symptoms, long duration of symptoms, female gender and young age were found to be weak predictors of poor outcome.

Level of evidence Case series, Level IV.

Keywords Elbow - Tennis elbow - Lateral epicondylitis · DASH $\cdot$ Outcome study $\cdot$ Predictor variables

\section{Introduction}

Tennis elbow (TE) —also called lateral epicondylitis, epicondylosis, epicondylalgia or tendinopathy-is a common disorder of the elbow with a prevalence of $1-3 \%$ in the general population and $7 \%$ in manual workers $[8,28]$. Previous studies have suggested a prevalence of $35-50 \%$ among tennis players $[8,28]$. However, a recent prospective study in junior tennis players reported elbow injuries in 9\% during the two studied years and found injuries to the ankle, shoulder or low back to be more common [18]. TE is occurring most often in the age group of 40-60 yearsexcept in tennis players who are generally younger-and it affects men and women to the same degree [8, 20, 28]. In addition to age, risk factors for developing tennis elbow include repetitive and forceful motions of wrist and arm, participating in racket sports, using a faulty tennis playing technique and smoking tobacco [8]. 
It has been claimed that conservative care leads to recovery in up to $90 \%$ of TE patients within 1-2 years and that surgery is indicated in less than $10 \%$ of the cases $[5,6$, 25]. However, recent studies of patients with elbow complaints, including $\mathrm{TE}$, in general practice report a less favourable prognosis [2, 17]. Bot and co-workers found that although $90 \%$ of all patients reported at least some improvement after 1 year of follow-up, only $13 \%$ of the patients reported full recovery at the 3-month follow-up and $34 \%$ at 12 months [2]. In patients with persisting pain and disability, surgery may be considered. Many different techniques have been described. However, at present no technique has been shown to lead to better results than the others. Few randomised studies have been reported, and many case studies are hampered by methodological shortcomings such as small study population, low percentage of follow-up and inclusion of cases with concomitant lesions in elbow, hand or shoulder [20]. The purpose of this study was to evaluate short-term (median 18 months after surgery) and medium-term (median 4 years after the surgery) results after open lateral release in recalcitrant tennis elbow and to determine any prognostic factors.

\section{Materials and methods}

Eighty-nine patients with 92 operated elbows met the criteria for inclusion in the study. Twelve patients did not agree to take part in the study. Thus, 77 patients (87\%), 38 male and 39 female, with 80 operated elbows were available for analyses. The median patient age at the time of surgery was 46 years (range, 34-64 years).

Patients with a characteristic history of activity-related pain at the lateral epicondyle interfering with the activities of daily living refractory to conservative care for at least 6 months and a confirmatory physical examination that included palpable tenderness over the extensor tendon insertion, provoked pain with resisted wrist and third digit extension, normal range of movement of the elbow, normal neurological status, normal ligamentous laxity tests and no radiographic joint derangement were included in the study. Exclusion criteria were restricted range of movement, neurologic deficits, ligamentous instability, previous surgery in the elbow, fracture sequelae, chondral or osteochondral lesions, osteoarthritis or loose bodies.

Evaluation of the results

Clinical outcome was evaluated by the 11-item disability/ symptom subset of Disabilities of the Arm, Shoulder and Hand Outcome Measure (DASH) named QuickDASH [1]. Data were collected before the operation and at the medians of 18 months (range, 6-36 months; short term) and 4 years (range,
3-6 years; medium term) postoperatively. We rated the QuickDASH outcome at the last follow-up as excellent $(<20$ points), good (20-39 points), fair (40-60 points) or poor ( $>60$ points) [26]. The patient's profession was classified as strenuous or non-strenuous with respect to the upper extremities [16].

\section{Surgical technique}

The surgery was carried out in an outpatient surgery unit in combined general anaesthesia and local anaesthesia subcutaneously with the patient placed supine with a standard technique similar to that described by Verhaar and coworkers [33]. A tourniquet was not used. A gently curved incision approximately four centimetres long was made directly over the lateral epicondyle. The extensor origin was exposed, divided transversely close to its attachment on the lateral epicondyle and allowed to retract distally [33]. The joint capsule was released along with the extensor origin, and a small incision was made through the synovial membrane allowing inspection of the joint [33]. Decortication of the bone at the attachment site at the lateral epicondyle was performed with an osteotome. The subcutaneous tissue and skin were sutured and a compression bandage applied.

\section{Rehabilitation}

The arm was rested in a sling for 2 weeks. Rehabilitation consisted of early active range of motion and eventual return to full activity as tolerated. Heavy or repetitive manual work was discouraged for 6 weeks.

\section{Statistical analyses}

The statistical analyses were made with the Statistical Package for the Social Sciences (SPSS Inc., Chicago, Illinois, USA) on a personal computer. As measures of central location and spread of data, mean and SD or median and range were calculated. Repeated measures one-way ANOVA was used to compare the QuickDASH scores at different points in time. Multiple regression was used to explore the relationship (regression model) between QuickDASH at the final followup (dependent variable) and a combination of possible predictor variables; baseline QuickDASH, sex, age, occurrence in dominant or non-dominant arm, duration of symptoms, sudden or gradual onset of symptoms, work-related cause, strenuous or non-strenuous work and/or occupation. A $P$ value $<0.05$ was considered significant.

\section{Results}

The median duration of symptoms was 13 months (range, 6-72 months). Fifty-five patients had experienced symptoms 
for 12 months or more. The onset of symptoms was sudden in 9 patients. Twenty-six patients considered their work as the main cause of the elbow problem, whereas 2 patients related their problem to sporting activities. None of the patients played tennis on a regular basis. Twenty-three patients had occupations classified as strenuous according to Haahr et al. [16].

Fifty-five right elbows and 25 left elbows were operated upon. The dominant elbow was involved in $71 \%$. We did not observe macroscopic ruptures or other convincingly grossly pathologic changes in the extensor origin or internal derangement of the joint such as chondral or osteochondral lesions, osteoarthritis or loose bodies. Major complications such as deep infection, permanent nerve injuries or stiffness of the elbow were not observed. Superficial wound problem/ infection was seen in three patients, and a postoperative haematoma was evacuated in one patient. In three patients, revision surgery was carried out due to lack of improvement during the observation period.

The mean QuickDASH was significantly improved compared with baseline both at the median 18-month and the median 4-year follow-ups (Table 1). No significant difference was found in mean QuickDASH between the short-term and the medium-term follow-ups. An improvement of the QuickDASH at the final follow-up compared with the baseline was observed in 78 of 80 (97.5\%) elbows. We rated the QuickDASH outcome according to Phillips et al. [26] as excellent in 58 of 80 elbows; good in 7 elbows, fair in 11 elbows and poor in 4 elbows.

We found a moderate correlation between the short-term and the medium-term results for the QuickDASH $(r=0.691 ; P<0.001)$. We found a weak correlation between the QuickDASH at the final follow-up (a high value denotes residual symptoms) and baseline QuickDASH ( $r=0.388 ; P<0.001$ ), acute occurrence of symptoms ( $r=0.362 ; P<0.001)$, duration of symptoms $(r=0.276$; $P=0.007)$, female gender of patient $(r=0.269$; $P=0.009)$ and age of patient $(r=-0.203 ; P=0.04)$. We found no significant correlation between the QuickDASH at the final follow-up and affection of dominant (vs. nondominant) arm, a work-related cause (as evaluated by the patient) or strenuous (vs. non-strenuous) work and/or occupation. Thus, the latter variables were excluded in the

Table 1 The mean value \pm SD of the QuickDASH [from 0 (best) to 100 (worst)] before the operation and at the short- and medium-term follow-ups

\begin{tabular}{lll}
\hline & QuickDASH & $P$ value* \\
\hline Preoperative & $61 \pm 16$ & \\
Median 18-month follow-up & $17 \pm 20$ & $P<0.001$ \\
Median 4-year follow-up & $18 \pm 19$ & $P<0.001$ \\
\hline
\end{tabular}

* In comparison with preoperative data stepwise regression analyses. The linear regression line equation was as follows: (QuickDASH at final followup) $=15.335+0.247$ (baseline QuickDASH) +17.845 (acute occurrence) +0.388 (duration) +4.057 (female gender) -0.440 (age) $(P<0.001)$. The overall model $R^{2}$ was 0.338 . The $P$ value for the regression was $<0.001$.

\section{Discussion}

Tennis elbow is generally believed to be caused by repetitive mechanical load of the elbow while using a forceful hand grip leading to an overuse injury of the extensor tendons insertion. The condition has often been called epicondylitis but histologic examinations have failed to demonstrate inflammatory cells. The pathogenesis is believed to be cumulative microtrauma exceeding the tissue's capacity for repair leading to a degenerative process characterised by disruption of tendon fibres, invasion of fibroblasts, disorganised collagen and vascular hyperplasia [5, 25]. Recent studies suggest the neovascularisation represents a healing response [4]. Macroscopically, the findings reported by different authors vary greatly and include little or no grossly pathologic findings [3, 30, 33]; 'greyish, immature scar tissue which appears shiny, oedematous and friable' [25]; and partial or total rupture of the extensor tendon origin [5]possibly reflecting different stages of a degenerative process. Swedish studies during the last decade have suggested that the pain in tennis elbow - as in achilles and patellar tendinosis-is caused by a so-called neurogenic inflammation mediated through neuropeptides such as substance-P and calcitonin gene regulated peptide [13, 23, 36].

Most surgical techniques aim to provide one or more of the following: relieve the stress at the tendons insertion by a release of the common extensor origin [19, 33]; removal of the degenerative tissue [25]; or stimulating repair by decortication of bone at the insertion site [25, 33]. A lateral release is performed by an open, mini-open or percutaneous approach. The latter approach may result in shorter rehabilitation [10].

The results after open surgery with recalcitrant TE have been reviewed by meta-analyses recently [20,24]. While the different studies constituting the meta-analyses cannot be directly compared, the surgical success rates for the open technique have been reported to be between 19 and $100 \%$ with a mean of $80.4 \%$ [20]. Thus, the results of our study$81 \%$ was rated as excellent or good at the final median 4-year follow-up_-seem to be in accordance with the typical outcome after open surgery. In the present study, the QuickDASH 11-item disability/symptom section of the original DASH questionnaire was used [1]. The QuickDASH is a more efficient version of the DASH outcome measure that appears to retain its measurement properties and can be 
used instead of the DASH with similar precision in upper extremity disorders [14]. The DASH or QuickDASH scores have been used for evaluating the outcome after surgical treatment of TE in several studies [10, 22, 32, 34].

Several modifications of the original technique described by Hohmann [19] have been suggested. Most commonly are removal of tissue with macroscopic degenerative changes and decortication of bone with an osteotome or by drilling [25]. In the present study, decortication by an osteotome of a small area of bone at the insertion site of the extensor origin was carried out. The rationale for decortication is the release of pluripotent stem cells that may accelerate the repair process. However, some studies suggest that decortication is not necessary for achieving repair [7] and that it may even hamper the outcome by resulting in more postoperative wound bleeding, stiffness and pain $[21,37]$. We observed only one case of postoperative hematoma requiring evacuation, but the design of our study does not permit any conclusion about the effect of decortication on the rate of complications or the outcome.

Similar to the observation by others $[9,33]$, we found little or no macroscopic degenerative changes in the extensor origin. However, as a transverse division of the extensor origin was performed only superficial changes will be visible, and deeper tendinous ruptures may be overlooked [25, 33]. We do not consider this to be a problem as the surgical technique used in our study does not include removal of tendinous tissue. In outcome studies with an observation period averaging 2 years or more, a good or excellent outcome has been reported in over $80 \%$ of the patients by both the Nirschl surgical technique or modifications of the latter that includes removal of degenerative tendinous tissue $[11,12,25]$ and the Hohmann extensor release without removal of degenerative tendinous tissue [27, 33]. Further, there is no correlation between the intensity of the histologic reaction and the clinical outcome [9]. At present, it has not been shown that removal of degenerative tendinous tissue results in an improved clinical outcome [20, 24].

The age (median, 46 years; range, 34-64 years), distribution of gender (38 male and 39 female) and percentage affection of dominant arm (71\%) of our patients are similar to that of other studies on surgical treatment of TE [25, 33]. The baseline symptoms and disability of our patients-as evaluated by the mean QuickDASH (61) — are also similar to that reported by others using the same set of questions [10, 22].

We found that high level of pain and disability at baseline ( $r=0.388 ; P<0.001$ ), acute occurrence of symptoms ( $r=0.362 ; \quad P<0.001)$, long duration of symptoms ( $r=0.276 ; P=0.007)$, female gender $(r=0.269 ; P=$ $0.009)$ and young age $(r=0.203 ; P=0.04)$ are weak predictors of poor outcome, i.e. a high QuickDASH. Unfortunately, few previous studies analyse possible predictors of poor (or good) outcome after TE surgery and it is difficult to find studies that support or refute our findings. High level of pain and disability at baseline [2, 15] and long duration of symptoms [2, 29] have previously been shown to predict worse outcome after conservative treatment of TE. Worse clinical outcome in women has previously been observed after both conservative care, including physiotherapy [2, 35] and surgery [31].

Many clinical studies on surgical treatment of TE are hampered by shortcomings including retrospective design, low number of patients, loss of patients to follow-up, shortterm follow-up period and inclusion of cases with concomitant procedures [20]. In evaluating the Coleman Methodology Score (CMS) of studies on operative management of tennis elbow, Karkhanis and co-workers [20] found that only 9 of 45 studies reported on more than 60 elbows (which is the lower study size limit for the top score of the CMS) and only five studies were sized 80 elbows or more. In the present study, 80 elbows (in 77 patients) were studied. We managed to follow-up $82 \%$ of the patients eligible for inclusion in the study.

Karkhanis found that the mean follow-up time fluctuated from 12 to 96 months in 45 outcome studies on surgical treatment of tennis elbow [20]. Whereas a long observation period is generally considered to strengthen the scientific value of a clinical study [20], most patients are just as interested in information about the short-term prognosis. Thus, it makes sense to include both a short-term follow-up and a medium- or long-term follow-up and to examine if a surgical result changes over time. In the present study, we evaluated the short-term (median 18 months) and mediumterm (median 4 years) results of the treatment of recalcitrant TE with release of the common extensor origin. Contrary to the results of Verhaar et al. [33] who found a clinical improvement from 1 to 5 years postoperatively, no significant difference in outcome between the short-term follow-up and the medium-term follow-up was demonstrated in our study. However, our first follow-up was done at a later point in time (medium 18 months) than in the study of Verhaar et al. [33].

The strengths [20] of our study include the high number of patients, more than $80 \%$ follow-up, a single uniform surgical technique, no concomitant lesions, both short- and mid-term follow-ups, the use of a patient administered outcome score and registration of baseline symptoms/disability. The important limitations of our study are the lack of a control group and functional testing, e.g. grip strength.

\section{Conclusion}

We conclude that open lateral extensor release performed as outpatient surgery results in improved clinical outcome 
at both short- and medium-term follow-ups with few complications. At the final follow-up (at median 4 years postoperatively), $81 \%$ was rated as excellent or good-a result that seems to be in accordance with the typical outcome after open surgery. High baseline disability, sudden occurrence of symptoms, long duration of symptoms, female gender and young age were found to be weak predictors of poor outcome.

Open Access This article is distributed under the terms of the Creative Commons Attribution Noncommercial License which permits any noncommercial use, distribution, and reproduction in any medium, provided the original author(s) and source are credited.

\section{References}

1. Beaton DE, Wright JG, Katz JN (2005) Development of the QuickDASH: comparison of three item-reduction approaches. J Bone Joint Surg Am 87:1038-1046

2. Bot SD, van der Waal JM, Terwee CB, van der Windt DA, Bouter LM, Dekker J (2005) Course and prognosis of elbow complaints: a cohort study in general practice. Ann Rheum Dis 64:1331-1336

3. Carter RM (1925) Epicondylitis. J Bone Joint Surg Am 7:553-562

4. Clarke AW, Ahmad M, Curtis M, Connell DA (2010) Lateral elbow tendinopathy: correlation of ultrasound findings with pain and functional disability. Am J Sports Med 38:1209-1214

5. Coonrad RW, Hooper WR (1973) Tennis elbow: its course, natural history, conservative and surgical management. $\mathrm{J}$ Bone Joint Surg Am 55:1177-1182

6. Cyriax J (1936) The pathology and treatment of tennis elbow. J Bone Joint Surg Am 18:921-940

7. Das D, Maffulli N (2002) Surgical management of tennis elbow. J Sports Med Phys Fitness 42:190-197

8. De Smedt T, de Jong A, Van Leemput W, Lieven D, Van Glabbeek F (2007) Lateral epicondylitis in tennis: update on aetiology, biomechanics and treatment. Br J Sports Med 41:816-819

9. Doran A, Gresham GA, Rushton N, Watson C (1990) Tennis elbow. A clinicopathologic study of 22 cases followed for 2 years. Acta Orthop Scand 61:535-538

10. Dunkow PD, Jatti M, Muddu BN (2004) A comparison of open and percutaneous techniques in the surgical treatment of tennis elbow. J Bone Joint Surg Br 86:701-704

11. Dunn JH, Kim JJ, Davis L, Nirschl RP (2008) Ten- to 14-year follow-up of the Nirschl surgical technique for lateral epicondylitis. Am J Sports Med 36:261-266

12. Dwyer AJ, Govindaswamy R, Elbouni T, Chambler AFW (2010) Are "knife and fork" good enough for day case surgery of resistant tennis elbow? Int Orthop 34:57-61

13. Eriksson E (2006) Is tennis elbow the same type of tendinosis as achilles and patellar tendinosis? Knee Surg Sports Traumatol Arthrosc 14:1037

14. Gummesson C, Ward MM, Atroshi I (2006) The shortened disabilities of the arm, shoulder and hand questionnaire (QuickDASH): validity and reliability based on responses within the full-length DASH. BMC Musculoskelet Disord 7:44

15. Haahr JP, Andersen JH (2003) Prognostic factors in lateral epicondylitis: a randomized trial with one-year follow-up in 266 new cases treated with minimal occupational intervention or the usual approach in general practice. Rheumatology (Oxford) 42:12161225
16. Haahr JP, Andersen JH (2003) Physical and psychosocial risk factors for lateral epicondylitis: a population based case-referent study. Occup Environ Med 60:322-329

17. Hay EM, Paterson SM, Lewis M, Hosie G, Croft P (1999) Pragmatic randomised controlled trial of local corticosteroid injection and naproxen for treatment of lateral epicondylitis of elbow in primary care. BMJ 319:964-968

18. Hjelm N, Werner S, Renstrom P (2010) Injury profile in junior tennis players: a prospective two year study. Knee Surg Sports Traumatol Arthrosc 18:845-850

19. Hohmann G (1933) Das Wesen und die Behandlung des sogenannten Tennisellenbogens. Münch Med Wochenschr 80:250-252

20. Karkhanis S, Frost A, Maffulli N (2008) Operative management of tennis elbow: a quantitative review. Br Med Bull 88:171-188

21. Khashaba A (2001) Nirschl tennis elbow release with or without drilling. Br J Sports Med 35:200-201

22. Knudsen R, Damborg F (2008) Surgical treatment of tennis elbow. Ugeskr Laeger 170:3328-3331

23. Ljung BO, Forsgren S, Friden J (1999) Substance P and calcitonin gene-related peptide expression at the extensor carpi radialis brevis muscle origin: implications for the etiology of tennis elbow. J Orthop Res 17:554-559

24. Lo MY, Safran MR (2007) Surgical treatment of lateral epicondylitis: a systematic review. Clin Orthop Relat Res 463:98-106

25. Nirschl RP, Pettrone FA (1979) Tennis elbow. The surgical treatment of lateral epicondylitis. J Bone Joint Surg Am 61:832-839

26. Phillips NJ, Ali A, Stanley D (2003) Treatment of primary degenerative arthritis of the elbow by ulnohumeral arthroplasty. A long-term follow-up. J Bone Joint Surg Br 85:347-350

27. Posch JN, Goldberg VM, Larrey R (1978) Extensor fasciotomy for tennis elbow: a long-term follow-up study. Clin Orthop Relat Res 135:179-182

28. Shiri R, Viikari-Juntura E, Varonen H, Heliövaara M (2006) Prevalence and determinants of lateral and medial epicondylitis: a population study. Am J Epidemiol 164:1065-1074

29. Smidt N, Lewis M, VDW DA, Hay EM, Bouter LM, Croft P (2006) Lateral epicondylitis in general practice: course and prognostic indicators of outcome. J Rheumatol 33:2053-2059

30. Spencer GE Jr, Herndon CH (1953) Surgical treatment of epicondylitis. J Bone Joint Surg Am 35:421-424

31. Svernlov B, Adolfsson L (2006) Outcome of release of the lateral extensor muscle origin for epicondylitis. Scand J Plast Reconstr Surg Hand Surg 40:161-165

32. Thornton SJ, Rogers JR, Prickett WD, Dunn WR, Allen AA, Hannafin JA (2005) Treatment of recalcitrant lateral epicondylitis with suture anchor repair. Am J Sports Med 33:1558-1564

33. Verhaar J, Walenkamp G, Kester A, van Mameren H, van der Linden T (1993) Lateral extensor release for tennis elbow. A prospective long-term follow-up study. J Bone Joint Surg Am 75:1034-1043

34. Wada T, Moriya T, Iba K, Ozasa Y, Sonoda T, Aoki M, Yamashita $\mathrm{T}$ (2009) Functional outcomes after arthroscopic treatment of lateral epicondylitis. J Orthop Sci 14:167-174

35. Waugh EJ, Jaglal SB, Davis AM, Tomlinson G, Verrier MC (2004) Factors associated with prognosis of lateral epicondylitis after 8 weeks of physical therapy. Arch Phys Med Rehabil 85:308-318

36. Zeisig E, Ohberg L, Alfredson H (2006) Sclerosing polidocanol injections in chronic painful tennis elbow-promising results in a pilot study. Knee Surg Sports Traumatol Arthrosc 14:1218-1224

37. Zingg PO, Schneeberger AG (2006) Debridement of extensors and drilling of the lateral epicondyle for tennis elbow: a retrospective follow-up study. J Shoulder Elbow Surg 15:347-350 